\title{
Multiversism and Concepts of Set: How much relativism is acceptable?*
}

\author{
Neil Barton ${ }^{\dagger}$
}

\begin{abstract}
Multiverse Views in set theory advocate the claim that there are many universes of sets, no-one of which is canonical, and have risen to prominence over the last few years. One motivating factor is that such positions are often argued to account very elegantly for technical practice. While there is much discussion of the technical aspects of these views, in this paper I analyse a radical form of Multiversism on largely philosophical grounds. Of particular importance will be an account of reference on the Multiversist conception, and the relativism that it implies. I argue that analysis of this central issue in the Philosophy of Mathematics indicates that Radical Multiversism must be algebraic, and cannot be viewed as an attempt to provide an account of reference without a softening of the position.
\end{abstract}

Keywords: Philosophy of mathematics, set theory, foundations of mathematics, multiverse

\section{Introduction}

The development of set theory since the 1930s has been partly shaped by a fascinating phenomenon: independence results. Since Gödel's proof of the consistency of the Axiom of Choice and Continuum Hypothesis with Zermelo-Fraenkel set theory, various model-theoretic techniques (notably the method of forcing developed by Cohen) have allowed us to show that for many sentences of set theory $\phi$, it is neither the case that $Z F C \vdash \phi$ nor $Z F C \vdash \neg \phi$. The case is particularly acute in set theory, as the kinds of statements that turn out to be independent from $Z F C$ are extremely natural questions, rather than gerrymandered statements of metalogic (such as the Gödel sentence for Peano Arithmetic). While the most infamous example is probably the Continuum Hypothesis, the phenomenon is visible with respect to a wide range of mathematical entities. So Hamkins writes:

"A large part of set theory over the past half-century has been about constructing as many different models of set theory as possible, often to exhibit precise features or to have specific relationships with other models. Would you like to live in a universe where $C H$ holds, but $\diamond$ fails? Or

*The author is very grateful to Ben Fairbairn, Joel Hamkins, Peter Koellner, Ian Rumfitt, and audiences in London and Milan for insightful and useful feedback on the issues discussed. Special mention must be made of Victoria Gitman, Alex Kocurek, Chris Scambler, and two anonymous referees whose detailed comments improved the paper immensely. The author also wishes to thank the Arts and Humanities Research Council for their support during the preparation of the paper.

${ }^{\dagger}$ Department of Philosophy, Birkbeck College, University of London, Malet Street, London, WC1E 7HX. E-mail: nbarto02@mail.bbk.ac.uk 
where $2^{\aleph_{n}}=\aleph_{n+2}$ for every natural number $n$ ? Would you like to have rigid Suslin trees? Would you like every Aronszajn tree to be special? Do you want a weakly compact cardinal $\kappa$ for which $\nabla_{\kappa}(R E G)$ fails? Set theorists build models to order." ([Hamkins, 2012b], p417)

There have been (broadly speaking) two reactions to this phenomenon. On the one hand, some regard these results as indicative of a failure of bivalence in set theory. Others, however, prefer to maintain that these sentences nonetheless have a definite truth value.

In this paper I am primarily concerned with one view that regards some sentences of set theory as non-bivalent. This is Joel David Hamkins' recently proposed and technically elegant 'Multiverse View', given in [Hamkins, 2012b]. I shall argue that Hamkins' position may be interpreted in one of two ways, either as giving an account of ontology and reference (with similarities to the ideas present in [Balaguer, 1998]), or alternatively as providing a framework for understanding set theory algebraically. Taking Hamkins' view in the former way, I suggest, leads to a referential regress: on a given occasion of purported set-theoretic reference there is a vicious non-well-founded dependency chain. A similar problem, I argue, can be pressed against Hamkins concerning various metalogical notions. Analysis of these problems reveals that if the Hamkinsian Multiversist wishes to have her view interpreted ontologically, she ${ }^{1}$ has to de-radicalise some of her theses. My strategy is as follows:

In section 1 I outline the version of Multiversism under consideration (given in [Hamkins, 2012b]) and some of its theoretical motivations. I note that there are at least two ways of interpreting his view: either as providing a philosophical view of ontology, or specifying an algebraic framework for the practice of set theory. On the former interpretation, I note that the method by which we refer to the subject matter of mathematics is usually through description, and is often argued to solve a problem present in [Benacerraf, 1973]. In section 2 I point out that this way of interpreting Hamkins' Multiverse View leads to a very strong form of relativism. I then use this to develop a regress, one which I argue is vicious. Further analysis reveals that if we wish to refer to mathematical objects in a philosophically satisfactory manner, a core of concepts must be taken to be determinately understood. Section 3 then examines some consequences of this relativism for the study of metalogic. It is argued that the inability of the Multiversist to provide a characterisation of finitude appears deeply problematic and is further evidence that if we are to maintain an account of reference, then certain mathematical notions must be kept absolute. In section 4 I argue that the algebraic interpretation does not suffer from these difficulties, but note that it does not provide an answer to Benacerraf's problem.

It is concluded that the Hamkinsian Multiversist must either soften her position to include some concepts as determinately understood, or alternatively take her view to be one not concerned with ontology and reference.

\section{The Radical Multiverse View}

To see from where the Multiverse View gains its motivation, it will be useful to first examine its polar opposite. Pre-theoretically, once we have accepted the Iterative

\footnotetext{
${ }^{1}$ In order to disambiguate, throughout this paper I speak as if the Hamkinsian Multiversist is female and her opponents are male (with the exception of Hamkins himself).
} 
Conception of Set as the conceptual underpinning of $Z F C$ set theory ${ }^{2}$ it is natural to hold the following view:

[Universism] There exists a single, unique, maximal, determinate universe of sets, to which we may refer precisely using our set-theoretic concepts (all of which, in turn are determinate ${ }^{3}$ ).

Universism thus ensures that every statement of set theory is determinately true or false, we have a determinate ontology and concepts that we can use to refer precisely to said ontology. The Multiversist takes it, however, that there are good reasons to reject Universism. In particular I will be concerned with a very radical form of Multiversism given in [Hamkins, 2012b]. A main argument of this paper will be that the position can be interpreted in different ways. For this reason, we begin with a coarse grained characterisation of his view and refine it later.

Explaining his philosophical standpoint, Hamkins writes:

"As a result [of the independence phenomenon], the fundamental objects of study in set theory have become the models of set theory, and set theorists move with agility from one model to another." ([Hamkins, 2012b], p418)

In order to arrive at a first approximation, I shall take this as an initial statement of Hamkins' view. He claims the fundamental objects of set-theoretic study are the models of different set theories. This characterisation immediately raises the following question: what theory should these models satisfy?

For the purposes of this paper, we shall take the models to satisfy first-order $Z F C$. There are two reasons for this choice. First, while Hamkins is potentially open to the consideration of different multiverses (such as the multiverse of second-order arithmetic), it is $Z F C$ set theory with which he is primarily interested. Second, the theory must be first-order as Hamkins holds that our interpretation of second-order variables requires a background set theory. For this reason, he holds that indeterminacy in first-order $Z F C$ would transfer to the second-order setting ${ }^{4}$

Thus, we arrive at the following initial characterisation of Hamkins' position:

[Hamkinsian Multiversism] There is no one universe of sets, but rather many. A universe of sets is simply a model of first-order $Z F C$.

A remark on terminology is important here. The Multiversism in question must be specified to be Hamkinsian, as there are many Multiverse views, each of which takes different concepts to be determinate. For example, a Zermelian form of Multiversism normally holds questions of 'height' (i.e. the ordinals that exist) to be indeterminate, but questions of 'width' (i.e. the subsets formed at $V_{\alpha+1}$ given some $V_{\alpha}$ ) to be determinate ${ }^{5}$ On such a picture the universes are the natural models

\footnotetext{
${ }^{2}$ We should be mindful here of the fact that the idea of the Iterative Conception underpinning $Z F C$ set theory is quite controversial in itself. There is an extensive literature on the topic, for a small selection see [Maddy, 1988] and [Potter, 2004]. I do not address the question further here, and take it as assumed that the Iterative Conception is the justificatory resource to which the Universist appeals.

${ }^{3}$ It should be noted that there is the scope to hold that there is just one Universe of sets but that it is indeterminate in some sense (say because, some of its properties are indeterminate). For just such a view, see [Feferman, 2011]. $\S 3$.

4 This fact will turn out to be important for assessing Hamkins' view, and is discussed in more detail in

${ }^{5}$ The idea has its conceptual roots in [Zermelo, 1930], but see also [Isaacson, 2011].
} 
of second-order ${ }^{6} Z F C_{2}$. Hence, questions concerning the low levels the hierarchy (such as $C H$ ) are determinate: the relevant $V_{\kappa}$ is the same in every natural model of $Z F C_{2}$. Many questions of height (such as the existence of an inaccessible cardinal) turn out to be indeterminate, as there are usually some models of $Z F C_{2}$ in which there are no cardinals of the required variety (for example, the smallest model of $Z F C_{2}$ does not contain an inaccessible). Conversely, we might hold a version of Multiversism on which questions of height are determinate, but questions of width (for example the Continuum Hypothesis) are indeterminate (such a view is advocated by [Steel, 2014]). Here, our universes would be the models which contain all the ordinals, including their inner models and (set) forcing extensions. Hamkinsian Multiversism represents a very extreme position in this regard, a universe on his view is simply any structure that satisfies first-order $Z F C$.

In order to understand what is at stake for the Hamkinsian Multiversist, I explain three motivations that have been given for the view: (1.) the generality of forcing constructions, (2.) the avoidance of arbitrariness, and (3.) the possibility of additional mathematical insight.

\subsection{The generality of forcing}

Universism, it might be argued, faces a substantial challenge in the form of the independence results. It is not simply the fact that there are statements that are independent (a fact that, though it requires explanation, may be dismissed as a side issue about the paucity of our proof-theoretic framework), but rather the manner in which these results are proved.

The issue is the following. Set-theoretic practice is replete with model-theoretic techniques that, given a particular model of set theory $\mathfrak{M}$, 'add' sets not already in $\mathfrak{M}$ to $\mathfrak{M}$. The clearest technique with which this is visible is forcing. In order to force over a particular model of set theory $\mathfrak{M}$ we begin with a partial order $\mathbb{P}=\left\langle P,<_{\mathbb{P}}, \mathbb{1}_{\mathbb{P}}\right\rangle \in \mathfrak{M}$. We then choose a filter $G$ on $\mathbb{P}$ that intersects every dense subset $D$ of $P$ that lies in $\mathfrak{M}$. Next, we add $G$ to $\mathfrak{M}$ and close under set-theoretic operations definable in $\mathfrak{M}$. The end result is a new model $\mathfrak{M}[G]$ that (given the correct choice of partial order) satisfies the axioms and the appropriate sentence for the independence proof. Importantly, however, $G \notin \mathfrak{M}$ for non-trivial forcing constructions 7 The challenge for the Universist lies in the fact that often set theorists will force using ' $V$ ' to denote the ground model. Normally, ' $V$ ' denotes the universe of all sets. Since the set added (call it ' $G$ ') cannot be in the model over which we are forcing, the Universist faces a problem. For, it appears that they should say that the statement "there is a forcing extension $V[G]$ such that..." is true: many forcing constructions make just this claim and the mathematics in question appears perfectly rigorous and consistent. However, while $G$ is a set, $G$ cannot be in $V$ by construction, but $V$ is supposed to be all the sets there are. It looks as though something has to give.

At this point, it should be noted that the Universist has several quite robust options involving complex simulations of the statements in question. He could, for example, use the Reflection Theorem in conjunction with the Downward LöwenheimSkolem Theorem to obtain a countable structure over which he can force 8 Alternatively, he could interpret the forcing via Boolean-valued models (or if she wishes

\footnotetext{
${ }^{6}$ This is only true, of course, if we are allowed to use the 'full' semantics for $Z F C_{2}$. See [Meadows, 2013] and |Koellner, 2013] for discussion of the surrounding philosophical issues.

${ }^{7}$ For details see [Kunen, 1980| and |Jech, 2002].

${ }^{8}$ In particular, this strategy seems attractive for the reason that often use of the term ' $V$ ' is patently an abuse of notation, designed to underscore the fact that any countable transitive model will suffice for the construction. See [Koellner, 2013] for discussion of some of these issues.
} 
to interpret the reasoning in a two-valued manner, use a Boolean ultrapower and quotient structure ${ }^{9}$. Still further, he could analyse the problematic statements syntactically using the forcing relation.

All this is satisfactory as far as it goes, and allows the Universist to interpret forcing talk where the symbol ' $V$ ' is used. The Multiversist rejoinder, however, is that it seems arbitrary to insist that $V$ does not have a forcing extension where the relevant notions seem perfectly well defined. The issues here are subtle and complex, and I defer consideration of Universist interpretations of forcing to different work. Suffice to note for current purposes that the Universist has to provide a complex and detailed paraphrase strategy in interpreting this set-theoretic discourse.

This is precisely what is not required under Hamkinsian Multiversism, however. For her, the account of set-theoretic forcing is as follows. We start with a particular set-theoretic background, that we take to be 'all' the sets and dub this model ' $V$ '. Then, when we perform the sorts of model-theoretic constructions that necessitate the addition of sets to the model, we simply move from $V$ to a different (equally legitimate) universe of sets that may (and usually does) satisfy very different sentences. We do not have to provide any awkward paraphrase, rather, we can hold that when a set theorist asserts statements about 'the universe' $V$ and moves to a forcing extension $V[G]$, she begins by considering some particular universe and then shifts reference to a different universe (i.e. the forcing extension). Where the Universist has to use a paraphrase strategy to interpret the claim " $V$ has a forcing extension $V[G]$ such that...", the Multiversist can simply assert that reference is entirely transparent: she begins by referring to a perfectly real and legitimate universe, and then simply shifts reference to an equally real and legitimate universe. In short, the Hamkinsian Multiversist contends that she avoids insisting in an ad hoc fashion that $V$ has no forcing extension, she can hold any construction involving the term ' $V$ ' to have perfectly good and transparent ontological reference.

\subsection{The avoidance of arbitrariness}

The forcing construction provides a clear example of where Universism has questions to answer in interpreting set-theoretic discourse. However, the problem is indicative of a wider issue in set theory:

"the most prominent phenomenon in set theory has been the discovery of a shocking diversity of set-theoretic possibilities. Our most powerful settheoretic tools, such as forcing, ultrapowers, and canonical inner models, are most naturally and directly understood as method of constructing alternative set-theoretic universes." ([Hamkins, 2012b], p418)

The point here is fairly simple: while forcing presents the most obvious conflict with Universism (in virtue of adding sets to the ground model) it nonetheless remains the case that there are many constructions that can be naturally understood from a Hamkinsian Multiversist perspective. Any particular $Z F C$-preserving construction can be interpreted as movement within the Multiverse. The Universist, in virtue of holding that there is a unique privileged Universe of set theory, has to explain why the seemingly perfectly natural set-theoretic constructions produce nothing more than model-theoretic representations within $V$ rather than bona fide universes. Again, the issues here are subtle and a full consideration is outside the scope of this paper ${ }^{10}$ However, we should note that the Hamkinsian sees it as a

\footnotetext{
${ }^{9}$ For details, see [Hamkins and Seabold, 2012].

${ }^{10}$ Again, some of these issues are given consideration in [Koellner, 2013].
} 
substantial theoretical virtue that she can interpret set theorists as concerned simply with other models that are fully legitimate universes in their own right, rather than having to provide a philosophical analysis of the difference between $V$ and these other structures.

\subsection{Additional mathematical insight}

The final reason I shall mention here is that (Hamkins claims) the Universist runs the risk of missing mathematical insights. He argues as follows:

"Such a perspective may be entirely self-consistent, and I am not arguing that the universe view is incoherent, but rather, my point is that if one regards all outer models of the universe as merely simulated inside it via complex formalisms, one may miss out on insights that could arise from the simpler philosophical attitude taking them as fully real." (|Hamkins, 2012b], p426)

The key point here is the following: model-theoretic constructions are no longer merely devices for showing relative consistency proofs (as was the case with Gödel and Cohen). Rather, such constructions are now used to show theorems about objects that are of interest in their own right (rather than say, a number-theoretic fact about proof codes). The emerging picture has been one in which the study of multiverses and interrelations between models (such as embedding properties between models and their forcing extensions ${ }^{11}$ have become of central importance in set theory. The Hamkinsian's philosophical position, she argues, facilitates this kind of thought: we can undergo the relevant constructions and study the models without worrying whether or not such talk can be 'simulated' in the context of $V$.

Again the issues here are philosophically subtle, and there is a burgeoning literature on the subject ${ }^{12}$ For the purposes of assessing the Hamkinsian on her own terms then, we may simply take the above three issues to be the motivations she would like to preserve in interpreting set theorists.

\section{The algebraic and ontological interpretations of Hamkin- sian Multiversism}

Let us take stock. We are now in a position where we have a coarse grained statement of a Hamkinsian position, and some considerations that motivate the view. In this section, I argue that further examination reveals two aspects to her view, one ontological and one algebraic. Later, I shall argue that the latter is the only tenable interpretation of Hamkinsian Multiversism.

\subsection{The ontological interpretation}

The ontological interpretation is perhaps the more intuitive way of understanding Hamkins' view. Here we regard the view as providing an account of ontology and reference. Such an interpretation is suggested by passages such as the following:

\footnotetext{
${ }^{11}$ See, for example, [Foreman, 2010].

${ }^{12}$ See, for example, [Koellner, 2013] and [Hamkins and Seabold, 2012].
} 
"I shall argue for a contrary [to Universism] position, the multiverse view, which holds that there are diverse distinct concepts of set, each instantiated in a corresponding set-theoretic universe, which exhibit diverse set-theoretic truths. Each such universe exists independently in the same Platonic sense that proponents of the universe view regard their universe to exist." ([Hamkins, 2012b], pp 416-417)

So, the picture offered is one on which each universe exists fully independently in reality, much as a Universist asserts the full and mind-independent existence of her $V{ }^{13}$ This provides us with an account of ontology, the multiverse (of $Z F C$ ) is constituted ${ }^{14}$ by models of first-order $Z F C$. While there may be practical or personal reasons for preferring one universe over another, no one universe is especially privileged ontologically.

Given that she now has a picture of ontology, it is incumbent upon the Hamkinsian to provide an account of epistemology. In particular, one would like to know how reference behaves, and its relationship to truth and knowledge.

The question of reference is important on this interpretation of Hamkinsian Multiversism. Crucially she wishes to hold that the study of set-theoretic properties consists in their behaviour within the Multiverse, and that questions of truth are substantiated but a real ontology. For example, Hamkins writes:

"The multiverse view is one of higher-order realism-Platonism about universes-and I defend it as a realist position asserting the actual existence of the alternative set-theoretic universes into which our mathematical tools have allowed us to glimpse." ([|Hamkins, 2012b], p417)

and

"set theorists [when performing model-theoretic constructions] move with agility from one model to another." ([Hamkins, 2012b], p418)

A natural way to understand claims of the above kind is through reference: when performing a model-theoretic construction, we start with some relevant universe $V$, and shift reference to a different $V^{\prime}$. How then does this reference to different universes within the multiverse occur? Hamkins is nowhere explicit, but does say the following:

"Often the clearest way to refer to a set concept is to describe the universe of sets in which it is instantiated, and in this article I shall simply identify a set concept with the model of set theory to which it gives rise." ([Hamkins, 2012b], p417)

\footnotetext{
${ }^{13}$ It should be noted that the analogy is not total. For instance, a Universist is likely to deny the existence of $V$ as a set for reasons of paradox. On Hamkins' view, however, any particular $V$ is a set in a taller model. Nonetheless, one can see the similarity, both assert that the subject matter of mathematics is constituted by mind-independent entities.

${ }^{14}$ Exactly which models of first-order $Z F C$ is a subtle issue, and one I shall not consider here. Hamkins specifies (in addition to his broad picture) a list of Multiverse Axioms, designed to axiomatise movement within the Multiverse. Of note is that (within a particular $V$ ) the collection of all models of $Z F C$ does not satisfy the Multiverse Axioms, though the collection of all computably saturated models of $Z F C$ does: see [Hamkins, 2012b] and [Gitman and Hamkins, 2011] for details. These subtleties are unimportant for the discussion here, no matter what the axioms taken to characterise the Multiverse are, the arguments carry over immediately.
} 
Thus we have a picture of reference on which each model is correlated with a set concept, and we refer this concept through a description. An immediate question is what expressive resources we may employ in providing such a description. Hamkins is very clear: any such description is first-order. The key issue here is that this account of reference to concepts, identified with models, via description results in a very tight link between understanding and reference to ontology. Regarding categoricity theorems, he says the following:

"The point is that a second-order categoricity argument, even just for the natural numbers, requires one to operate in a context with a background concept of set. And so although it may seem that saying "1, 2, 3, . . and so on," has to do only with a highly absolute concept of finite number, the fact that the structure of the finite numbers is uniquely determined depends on our much murkier understanding of which subsets of the natural numbers exist." ([Hamkins, 2012b], p428)

Here, we see Hamkins argue that indeterminacy in the object language transfers to the metalanguage: if we are unable to pin down objects using our first-order set theory, and our second-order ${ }^{15}$ theories are dependent upon an understanding of the former, then a second-order metalanguage is also indeterminate. Hence, for the Hamkinsian, we are always confined to first-order descriptions ${ }^{16}$

Could the Hamkinsian hold the metalanguage fixed? While there is no logical contradiction in doing so, we shall assume that she accepts indeterminacy in her metalanguage (and indeed metametalanguage et cetera). There are two reasons for this choice. First, we are interested in Multiversism in its most radical form, and so would like to examine the case where the metalanguage is also indeterminate. Second, to hold the metalanguage determinate seems anathema to the Hamkinsian's dialectical position with respect to the Universist. Recall that some of the main motivations for rejecting the Universist position were his acceptance of seemingly ad hoc interpretations of model-theoretic constructions (especially forcing) for creating alternative set-theoretic universes. An insistence on the determinacy of the metalanguage when the object language is taken to be highly non-absolute would thus seem like her own set of $a d$ hoc assumptions. This is especially so given that the standard way of characterising the metalanguage is in set theory itself. Further, and more conclusively, if the Hamkinsian were to baldly assert determinateness of the metalanguage, it seems that her opponent would be perfectly entitled to likewise claim that his understanding of various set-theoretic concepts are determinate (such as the power set operation) opening the door to categoricity arguments and a rejection of Hamkinsian Multiversism.

We can then see that talk of moving from one universe to another cannot be, strictly speaking, what is occurring on a given occasion of set-theoretic reference. For, by several metatheoretic results, first-order theories are completely unable to precisely determine their objects up to isomorphism ${ }^{17}$ Hence, we lack the conceptual resources to pin down a single universe precisely. We do not, therefore, literally 'pick out' a model, perform a construction on said model, and move to a different

\footnotetext{
${ }^{15} \mathrm{Or}$, indeed, any substantial increase in expressive resources in the metalanguage (e.g. ancestral logic).

${ }^{16}$ This is not to say that second-order set theory is meaningless for the Hamkinsian. Rather, the interpretation of the second-order variables is dependent upon background concept of set, and so indeterminacy in the first-order language of $Z F C$ carries over to the second-order language.

${ }^{17}$ For example, the Löwenheim-Skolem Theorems entail that if a theory has an infinite model, then it has (clearly non-isomorphic) models of every infinite cardinality. The existence of non-well-founded models (an immediate consequence of the Compactness Theorem for first-order theories) is another good example of clearly non-isomorphic models of theories that are the same with respect to first-order satisfaction.
} 
model: we lack sufficient and determinate conceptual resources to accomplish such a task. One way to understand Hamkins' suggestion is to hold that we refer to several universes at once via description ${ }^{18}$ Since second-order resources are indeterminate as to interpretation of the variables, these descriptions are first-order, and as such do not specify any one universe precisely. Set-theoretic reference within the multiverse should thus not be thought of as referring to a particular set-theoretic possibility and then moving from this via a construction, but rather identifying a 'cloud' of universes within the multiverse all of which satisfy some particular sentences of first-order set theory. We then perform a model-theoretic construction, relative to each model in the cloud, which shifts focus to a different cloud of universes within the multiverse. We can then still hold on to our understanding of the practice of set theory, it is just that ' $V$ ' refers to some (contextually fixed) collection of universes, rather than a single one, and construction is relative to each background. This then moves us to a different cloud of universes satisfying different sentences.

Similar views receive currency in the philosophical literature, and have been seen to solve philosophical problems. For example [Balaguer, 1998] sketches a similar philosophical view (his affinity with which Hamkins notes ${ }^{19}$ ) on which any coherent first-order description correctly describes part of mathematical reality. In particular Balaguer sees his view as solving the following classic philosophical problem with objects-based realist accounts:

[Benacerraf, 1973] (Benacerraf's Problem) How can we refer to mathematical objects at all if we cannot come into direct epistemic contact with them?

Of course, the exact exegesis to be given to this problem is a thorny philosophical issue in itself. Benacerraf's original statement relies explicitly on a causal theory of knowledge, an account few would now accept. However, we may take the problem as well posed: given an acceptance of realism and the existence of mathematical objects as mind-independent, aspatio-temporal entities, how do we gain knowledge of such objects?

The question is of particular interest for the Hamkinsian, as it presents a possible advantage over the Universist. While Universists have proposed various solutions to the problem, either by denying it is actually a problem ([Maddy, 2011]), providing a categoricity argument ([McGee, 1997], [Martin, 2001]), or positing a Gödelian faculty of intuition ([Gödel, 1947]), all are fraught with controversy. If she can motivate the claim that she has a solution to the above problem, then this would place her in a far stronger dialectical position.

Balaguer sees his view as responding to the following characterisation of Benacerraf's problem:

"in order to salvage their view, mathematical platonists have to explain how human beings can acquire knowledge of abstract mathematical objects, given that they are not capable of coming into any sort of contact with such objects, that is, receiving any information from such objects." ([Balaguer, 1998], p48)

To which he responds as follows:

\footnotetext{
${ }^{18}$ It should be noted that he states that this is only 'often' how reference is achieved. However, nowhere else is he explicit about exactly how reference occurs.

${ }^{19} \mathrm{See}$, for example, his presentation in [Hamkins, 2012a].
} 
"Thus, in connection with the epistemological problem, the point is this: if I know that some theory truly describes part of the mathematical realm, then I have knowledge of that realm, regardless of whether it describes a unique part of that realm" ([Balaguer, 1998], p50)

The similarity with Hamkins' view is clear: we have first-order descriptions that (non-uniquely) identify part of the mathematical realm. Balaguer and Hamkins' positions are thus similar in content whilst motivated by different considerations. Balaguer attacks an epistemological problem concerning knowledge of mathematical objects in light of Benacerraf's Problem, whereas Hamkins is motivated by worries (both epistemological and ontological) arising from the vast proliferation of settheoretic models. Common to both the ontological interpretation of Hamkinsian Multiversism and Balaguer's view is that knowledge of mathematical objects can be accounted for by understanding first-order reference to apply to part of the mathematical realm.

Comparison of the ontological interpretation of Hamkinsian Multiversism and Universism is thus particularly interesting from a philosophical perspective, because both are paradigms of realism. Each seeks to underpin mathematical practice and truth by having sets be abstract objects in ontologically good standing. However, both views have very different conceptions of the nature of the subject matter of set theory, a fact which is manifest in their attitudes to the bivalence of statements such as the Continuum Hypothesis. The Universist should think that $\mathrm{CH}$ has a determinate truth value, holding as they do that there is a single determinate maximal universe of sets to which we may precisely refer. However for the Multiversist there is no fact of the matter whether or not $C H$ is true, some set-theoretic backgrounds that satisfy $Z F C$ also satisfy $C H$, and some do not. In particular any universe $V$ has a forcing extension $V[G]$ (in which no cardinals are collapsed) such that $V[G] \models Z F C+\neg C H$ and a different extension $V[H]$ (adding no new reals) such that $V[H]=Z F C+C H$. Asking "Is $C H$ true?" is comparable to asking whether the Parallels Postulate is true in geometry: just as the Parallels Postulate depends on with which geometry one is concerned, so $\mathrm{CH}$ depends on the particular set-theoretic background under consideration.

\subsection{The algebraic interpretation}

The ontological intepretation is not, however, the only way of interpreting Hamkins' view. We begin by noting a difference between two kinds of mathematics. On the one hand we have particular mathematics such as number theory and analysis, where we (at least prima facie) aim to talk about some particular structure. On the other hand, we have algebraic mathematics, on which we explicitly are not concerned with any particular objects, rather we examine what will be possible on a given structure with certain properties.

To see the distinction more clearly, consider a paradigm case of particular mathematics such as number theory. Here we aim to talk about properties held by individual numbers and the natural number structure. For example, we can claim that the number 7 is prime, and in so doing make a claim about properties held by a particular position in the structure $\mathbb{N}$. On the other hand, a paradigm example of algebraic mathematics is group theory, where we study the basic properties of any structure satisfying the group axioms (and possibly extensions thereof). We are not interested in what the objects are, rather we concern ourselves with what will hold and can be constructed given that we are presented with a structure with certain properties. 
Hamkins, however, occasionally argues that on his view set theory is more of a piece with disciplines such as group theory:

"While group theorists study groups, ring theorists study rings and topologists study topological spaces, set theorists study the models of set theory." ([Hamkins, 2012b], p418)

How should we understand the practice of algebraic mathematics? It is tempting to understand it in a similar way to the ontological interpretation of Hamkinsian Multiversism: by providing descriptions of what it takes to be a group or a ring we might be said to describe part of the mathematical realm. Certainly, one could take this view of algebraic mathematics, and in doing so would collapse the algebraic interpretation of Hamkinsian Multiversism into the ontological interpretation. However, especially as the end argument will be that the ontological interpretation is philosophically incoherent, we shall see if there is an alternative understanding available.

A different way to interpret algebraic mathematics such as group theory views the disciplines not as concerned with description and reference, but rather axiomatising facts about what holds and can be constructed relative to certain kinds of mathematical structure. In the case of group theory, if we take a group $G$, we are able to investigate (a) what holds on $G$, and (b) what we can construct from $G$. For example, we know that if $G$ has a prime number of elements, then $G$ is cyclic. Further, we can construct new objects from $G$. Taking quotients, direct products, semidirect products, wreath products, direct limits, inverse limits et cetera are most naturally understood as methods of constructing new groups from old. We can understand these operations not as making any claims about existence and reference, but rather telling us what will happen given some objects endowed with some operations and relations.

The idea then for the Hamkinsian is to hold that her view is not concerned with existence and reference but rather explaining what will hold and can be constructed given some structure that satisfies $Z F C$. We do not make any claims as to what exists within the Multiverse, rather it is seen as an intuitive picture to facilitate algebraic reasoning concerning sets. Given a structure, the Multiverse View tells us how we can move from this structure. Indeed it is natural to view some of the technical achievements of the Multiversist programme in this light. For example, we can understand the project of providing the modal logic of forcing in [Hamkins and Loewe, 2008] as an attempt to explain how we can move from a ground model using forcing constructions.

It should be noted that prima facie the two interpretations are not in conflict with one another. Indeed, it is very natural to adjoin the algebraic interpretation to the ontological interpretation in providing an account of exactly what the Hamkinsian is advocating concerning set-theoretic practice. Despite this, the rest of the paper will argue that the ontological interpretation is unsatisfactory (without a softening of the position). Nonetheless, we shall see that the algebraic understanding of the Hamkinsian is not thereby threatened.

\section{Relativism and the referential regress}

Let us return then to the ontological interpretation of Hamkinsian Multiversism. Recall that on this interpretation we refer via description to a cloud of universes within the Multiverse. 
We should first note that Hamkins is committed to a very strong form of relativism: what sets exist and the kinds of model-theoretic constructions possible are relative to a particular initial set-theoretic background. Given this, the extent of the multiverse is also indeterminate until a particular point is chosen. This is a fact of which Hamkins is well aware:

"although it is better to understand these descriptions [forcing et cetera] as relative construction methods, since the resulting universe described depends on the initial universe in which the constructions are undertaken...one does not expect the properties of the multiverse to be available when undertaking an internal construction within a universe. That is, we do not expect to see the whole multiverse from within any particular universe." ([Hamkins, 2012b], p417)

That is to say, the way the Multiverse itself appears differs according to the universe in which one starts. One immediate response to the Hamkinsian would then be the following argument. We might note that, as the extent of the multiverse is determined by set-theoretic background, the following statement is true:

"We cannot quantify over the whole multiverse."

Now, this particular statement is clearly true for Hamkins. Let 'extent $V_{1}$ of the multiverse' denote the extent of the multiverse from the point of view of $V_{1}$. We know that for any extent $V_{V_{1}}$ of the multiverse, there is a different extent $V_{V_{2}}$ of the multiverse that contains universes not in the extent $V_{1}$ of the multiverse. Thus there are always universes in 'the whole multiverse' over which we cannot quantify given the fixing of some background concept of set $V$. The statement is, at first blush, somewhat paradoxical: it violates its own content by quantifying over the whole multiverse.

We should note, however, that such a paradox is merely intuitive and informal. Since there is no known system in which the notion of the whole Multiverse is formalisable, claims about the Multiverse are formulated in terms of set models within some initial starting background $V$. Thus, with respect to formal analysis, there is no direct contradiction. For, within some $V$ we can perfectly well quantify over 'the whole Multiverse': $V$ simply takes the Multiverse to be some models satisfying the Multiverse Axioms. Given the lack of direct contradiction, we might question the extent to which the Hamkinsian Multiversist will find such arguments convincing. Further, such relativistic paradoxes are well worn in the literature on absolute generality. I will not, therefore, re-examine those issues here. However, I will press a different worry on exactly how the Multiversist is able to secure reference to any particular universe within an extent $V$ of the multiverse.

We begin by observing that the model-theoretic constructions possible are dependent upon the particular background of set in which one finds oneself, let it be denoted by ' $V_{1}$ '. Thus $V_{1}$ determines the extent $V_{1}$ of the multiverse. Given this, the exact cloud $c_{1}$ picked out by a given utterance of set-theoretic statements is in fact dependent on $V_{1}$ : if the extent of the multiverse had been different, we would have picked out a different cloud. But as it was acknowledged earlier, picking out an exact universe $\left(V_{1}\right)$ is impossible as we are restricted to referring to models using concepts expressed as first-order axiomatisations. Thus it turns out that the initial selection of $c_{1}$ was dependent on a prior selection of a different cloud $c_{2}$. Again though, selection of $c_{2}$ is dependent on the extent of the multiverse, and hence on selection of a different universe $V_{2}$ to determine the extent $V_{2}$ of the multiverse. But reference to $V_{2}$ 
is impossible, and hence this should really be analysed as reference to some cloud $c_{3}$. It is clear that there is no end to this process, and we have a non-well-founded dependency chain.

Clearly a referential regress looms. Our reference depends upon an infinite descending sequence of cloud and universe specifications. Normally (though not always), in philosophical discussion, the production of a regress in a situation where one would expect grounding (such as with respect to reference) is sufficient to show that the view faces some serious difficulties. However, it is one thing to show that a regress looms, and another to show that it is vicious in nature. This is particularly so for the case at hand as, by and large, the Multiversist is happy with the idea of non-well-foundedness. Indeed, a central tenet of the Multiverse View states that every universe is non-well-founded from the perspective of a different universe ${ }^{20}$ So, is the regress vicious?

One might think that it is not, in fact, a vicious regress. While one might worry that in the case of reference a non-well-founded dependency structure is unacceptable, there are examples that show that this is not implausible. Imagine, for example, a non-actual, non-atomic, possible world composed entirely of ever smaller particles of increasing fundamentality. Let ' $o_{1}$ ' denote some object in this world. One might think that we successfully refer to $o_{1}$ in virtue of successfully referring to all its smaller parts of the next level of greater fundamentality $o_{2}^{\prime}, o_{2}^{\prime \prime}, o_{2}^{\prime \prime \prime}, \ldots$ We might then think that we refer to these in virtue of referring to all their parts of the next level of fundamentality $o_{3}^{\prime}, o_{3}^{\prime \prime}, o_{3}^{\prime \prime \prime}, \ldots$ and so on. Here, the non-well-founded dependency structure of reference is not obviously vicious.

However, epistemological considerations tell us that in the case of the Multiversist it is indeed a vicious regress. Recall the Multiversist's position regarding the link between ontology and concepts. There it was noted that the Multiversist thought that every model of set theory constituted a set concept. Now, it was then noted that, by the Multiversist's own lights, this could not quite be the correct story. The impossibility of singling out individual set-theoretic backgrounds meant that it was far more accurate to say that a set concept is related to a particular cloud of universes within the multiverse. However, now it has been shown that such an occasion of reference requires picking out infinitely many such clouds. The problem is now very serious: given that there is a regress it seems that we are employing infinitely many concepts on a given occasion of reference. The idea that we could possess infinitely many concepts is highly controversial (if not downright absurd), let alone employ infinitely many concepts in referring. The situation in the non-atomic world is substantially disanalagous: there we were only using one concept to refer (the concept that sufficed to pick out $o_{1}$ ), it just happened to be the case that this reference had a non-well-founded dependency chain.

Is there a way for the Multiversist to modify their view such that they can avoid this problem? I see two main ways of achieving this goal:

1. Modify her view so that the regress is no longer vicious.

2. Modify her view in such a way as to prevent the generation of the regress at all.

What are the prospects for the first option? If we analyse the non-atomic universe, we can see that the need for infinitely many concepts avoided in virtue of the fact that the speaker at the world did not need to possess the concepts for the more fundamental objects in order to secure reference: in this case reference to $o_{1}$ was

\footnotetext{
${ }^{20}$ This is known as the Well-foundedness Mirage. See [Hamkins, 2012b], p439.
} 
achieved via direct contact with it and all its parts of greater fundamentality. The need for infinitely many concepts is precisely what is required for the Multiversist, in order to know about which cloud we are talking, we must first know what other clouds we are fixing in order to secure reference to the initial cloud, and every such cloud corresponds to a concept. The Hamkinsian could then possibly avoid the viciousness of the regress by denying the link between concepts and ontology: they could deny that every cloud corresponds to a set concept.

This response is deeply problematic for familiar reasons in the philosophy of mathematics. In the case of the non-atomic universe, the speaker has direct contact with objects of the kind to which she is referring. This is precisely not so with the proposed account of reference in higher set theory: the way we refer to these entities is entirely through description. If we deny that we have a concept for referring to each cloud, there would be a point at which we did not have a fixed background, and hence reference would break down. Denying the link between concepts and ontology undercuts the Multiversist's initial starting point for reference.

The second option then looks slightly more promising: we should modify the Multiverse View to prevent the regress getting going at all. Recall that the catalyst for the regress was the fact that the extent of the Multiverse itself was relative, this then infected our reference to any particular cloud. Two responses present themselves: either we can argue that this sequence of reference nonetheless has a stopping point despite the indeterminacy in the extent of the Multiverse, or alternatively prevent the regress by delimiting absolutely the extent of the Multiverse.

How could we accomplish the former? Instead of maintaining that the extent of the Multiverse is dependent on where we start, she could instead say that its extent is indeterminate in virtue of being so dependent. On a given occasion of set-theoretic reference, while there may be a potential regress, we simply do stop somewhere, it is just that we do not know where we have stopped. The Multiverse is then fixed from this particular stopping point, which may be different on any particular occasion.

Certainly, there is no contradiction in asserting this. There is nothing that she has said that prevents her from maintaining that we simply end up referring to a particular universe to act as our reference frame for talking about the Multiverse. Such a response is, nonetheless, highly dialectically ineffective. We should be mindful here of the Hamkinsian's complaint against the Universist that he is ad hoc in his disavowal of the existence of legitimate universes other than $V$. For, the Hamkinsian can give no particular reason to focus on one stopping point rather than another (nor any determinately understood language in which we could try to differentiate stopping points). This process of stopping at some $V$ would thus be to admit her own ad hoc assumptions. Further, such a response would undermine the purported advantage the Multiversist wishes to establish over the Universist concerning Benacerraf's Problem. Salient here is the fact that the Hamkinsian sees herself as providing a response to this central issue by accounting for reference through description without positing the existence of non-natural mental powers (as often Gödel is accused of doing). However, the response that we simply stop somewhere (without being able to give any reason for a particular stopping point) seems, like Gödel, to ascribe unexplained powers to the human mind.

Thus a preferable option is to be able to genuinely single out particular settheoretic backgrounds as more privileged than others, thereby securing the nonrelativity of the model-theoretic constructions. In order to do this, we must have some model or models privileged within the multiverse, such that the key modeltheoretic constructions are absolute with respect to these models. In turn, to facilitate this, we require a stock of absolutely understood concepts, sufficiently rich in 
character that we can identify determinately a class of set-theoretic backgrounds.

The key point to take away from the discussion is the following: in any philosophy of mathematics that tries to make sense of a multiverse picture of set theory as concerned with reference, there is likely to be an element of relativism of certain concepts. However, there is a limit to how far this relativism can go. In particular we need to be precise about which universes (off the bat) are to count as privileged, and in doing so will have to specify a list of concepts that we are simply taking to have absolute significance in order to restrict our class of models. Moreover, this stock of concepts must be sufficiently rich to allow the relevant model-theoretic techniques to be absolutely specified, and the multiverse given precise limit. The acceptance of 'any particular' first-order model as a legitimate universe results in a relativism so strong that it cripples our ability to refer at all.

\section{Relativism and metalogic}

Consequences of this relativism can be pushed further to create more problems for the Multiversist. Due to the centrality of the claim that no-one universe of sets is more ontologically privileged than another for the Hamkinsian, several key concepts that many would take to be determinate turn out to not be so. Two good examples are the following ${ }^{21}$

Well-foundedness Mirage. Every universe $V$ is non-well-founded from the perspective of another universe $W$.

Countability Principle. Every universe $V$ is countable from the perspective of another universe $W$.

Why do the above principles hold? The Well-foundedness Mirage follows from several metatheoretic results: we know that there are models of $Z F C$ on which the 'membership relation' is non-well-founded from the perspective of the 'standard' model ${ }^{22}$ Many of these models will be able to see (from the perspective of their 'membership relation') a descending 'membership' sequence in what we currently take to be the 'standard' model, and hence view the current model as non-wellfounded ${ }^{23}$ In turn the Countability Principle holds because any cardinal can be collapsed to $\aleph_{0}$ using forcing arguments.

The ramifications for the Multiverse View are both striking and immediate. Any properties that are not absolute between all models of $Z F C$ turn out to be inherently dependent on our background concept of set. In this sense, the Multiversist's relativism about set-theoretic background extends to a relativism about many concepts ordinarily taken to be well understood. This is certainly controversial, but a feature of the view Hamkins is happy to embrace: he feels it makes sense of our experience with the diverse the set-theoretic possibilities provided by the model-theoretic constructions. However, we should be mindful of exactly how far this relativism goes. In particular, the relativism even applies to our understanding of natural numbers. Hamkins says the following:

\footnotetext{
${ }^{21}$ [Hamkins, 2012b], pp438-439.

22 Examples of this sort include the Mostowski Collapse Lemma, Compactness Theorem for first-order theories, and Ultrapower Construction (the former implies the existence of non-well-founded models indirectly, the latter two by explicit construction).

${ }^{23}$ The astute reader will notice that the explanation given here is riddled with 'scare quotes'. There is good reason for this, even the notion of standardness itself is relative for the Hamkinsian Multiversist.
} 


\begin{abstract}
"So why are mathematicians so confident that there is an absolute concept of finite natural number, independent of any set-theoretic concerns, when all of our categoricity arguments are explicitly set-theoretic and require one to commit to a background concept of set? My long-term expectation is that technical developments will eventually arise that provide a forcing analogue for arithmetic, allowing us to modify diverse models of arithmetic in a fundamental and flexible way, just as we now modify models of set theory by forcing, and this development will challenge our confidence in the uniqueness of the natural number structure, just as set-theoretic forcing has challenged our confidence in a unique absolute set-theoretic universe." ([Hamkins, 2012b], p428)
\end{abstract}

Here we see Hamkins express doubts that there is any determinate concept of natural number, on the grounds that any attempt to determine the structure of the natural numbers up to isomorphism will have to use resources beyond first-order, and hence will be dependent on the background notion of set. This lack of confidence in an absolute concept of natural number, though it brings out another controversial aspect of the view, is fine as far as it goes. However, the thesis that the natural numbers are a relative concept points to a familiar objection to views that hold that purely first-order theories are the only legitimate expressive resources. We begin with the following well-known theorem:

Theorem 1. Let $S$ be a set of first-order sentences and let $\phi(x)$ be any first-order formula containing only $x$ free. If, for every natural number $n$, there is a model of $S$ in which the extension of $\phi$ contains at least $n$-many objects, then there is a model of $S$ in which the extension of $\phi$ contains infinitely-many objects.

The theorem (a quick consequence of the Compactness Theorem) has the following immediate consequence: no purely first-order theory can ever pin down the notion of finiteness up to isomorphism ${ }^{24}$ Now real problems are beginning to emerge for the Multiversist. For, the formal framework of her view is to study the Multiverse through analysing the models of $Z F C$. Hence, she would like there to be a well-understood and determinate notion of proof and well-formed formula. Without these, the notions of a proof in $Z F C$ and indeed $Z F C$ itself are not even determinate. It is therefore indeterminate what the Multiversist is even asserting in saying that universes are models of $Z F C$, and her formal framework breaks down.

Again, the Hamkinsian could respond by arguing that there is a determinate notion of natural number and finitude relative to an initial starting $V$. Every $V$ thinks that it has the standard natural numbers and well-formed formulae. The relevant model-theoretic constructions thus go through relative to that background. Later, we shall see that the intuition behind this thought helps to clarify the algebraic interpretation. For the moment, however, this response falls flat on the ontological interpretation. There we are concerned with attempting to describe and refer to part

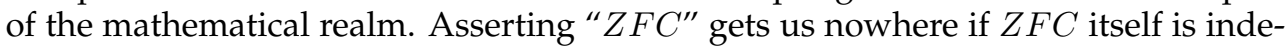
terminate: it fails to tell us anything about the way mathematical reality is as it is not clear what is being asserted.

Again, we might take this as indicative of the fact that certain notions need to be taken to be absolute. It has long been noted that certain mathematical concepts

${ }^{24}$ Many of these considerations, and in particular the above theorem, are discussed in Shapiro's seminal [Shapiro, 1991]. For a discussion of the similarities and differences between indeterminacy in the notion of finiteness and the explicitly set-theoretic case, the reader is directed to [Field, 2003]. 
are necessary for the expression of metalogical definitions ${ }^{25}$ By adhering to a very strong form of relativism, the Multiversist undercuts the very concepts required to properly express her own view.

\section{Giving up on the Benacerrafian Challenge: a response for the Hamkinsian Multiversist through the algebraic interpretation}

We have seen two problems for the ontological interpretation of the Hamkinsian Multiversist, one concerning a referential regress and a second concerning difficulties with metalogic. A solution was outlined: soften the radical nature of the view and take some notions to be determinately understood. I want to now consider a different approach: reject the ontological interpretation and simply continue with the algebraic interpretation. This will, however, force her to give up her view as a response to Benacerraf's Problem.

The solution in question involves rejecting that Hamkins' view should be construed as concerned with ontology and reference, and rather view set theory as an algebraic enterprise. Recall the options presented for avoiding the referential regress:

1. Modify the view so that the regress is no longer vicious.

2. Modify the view in such a way as to prevent the generation of the regress at all.

We can take option (2.), but rather than trying to maintain an account of reference, simply give up on set theory as the kind of enterprise in which we try to refer. Recall our paradigmatically algebraic discipline: group theory. As noted earlier, when reasoning with group theory we do not take ourselves to be referring to any particular group, rather we are simply say what will hold and be possible on any given structure that satisfies the group axioms.

We can view Hamkins' project in a similar light. Rather than taking him to be solving a problem of reference through description, we can take him to be telling us what will be satisfied and possible on any structure that satisfies the $Z F C$ axioms. We make no claim as to the universes that exist, and indeed cannot speak about the extent of or situate ourselves within the Multiverse. We can, however, say that given some structure $V$ that satisfies $Z F C$ certain things will hold and can be constructed relative to $V$. It is then simply a bad question to ask which universe we are in or what universes there are, much as asking which exact objects one is talking about in group theory is misguided.

How does this response solve the earlier problems? With respect to metalogic, it is true that on the algebraic conception we do not have a determinate understanding of natural number, finiteness, well-formed formula, or proof, independent of set-theoretic background. For, while any background $V$ in which we find ourselves provably holds itself to be standard (with the standard natural numbers and the standard well-formed formulae and proofs) we can easily construct a universe that views $V$ as non-standard. The important point from the perspective of the working set theorist is that any proofs or well-formed formulae they talk about will have the normal properties relative to any particular universe of sets in which they may find themselves, and hence the normal constructions will be possible from that background. In

\footnotetext{
${ }^{25}$ Certainly, at least since, Shapiro, 1991.
} 
this sense, the attempted response on behalf of the Hamkinsian in the previous section was on the right track. However, by holding that her view makes claims about an ontology to be described, the Hamkinsian Multiversist found herself in trouble, she then needed $Z F C$ to have determinate content in order to say something meaningful about the mathematical reality. Here, since our claims are already explicitly relative, there is no such problem. We are not making any claim about mathematical reality, rather we simply state what can be done from within a particular structure.

Moreover, the issue of reference is dealt with very trivially. Under the algebraic interpretation, we do not take set theory as the sort of enterprise that is concerned with referring to objects. Rather it is seen as providing an intuitive framework that underlies an algebraic method of thinking. This then allows us to understand what will be possible on a given structure with certain properties. Thus the problem of reference dissolves: we are not even making the appropriate kinds of claims to be assessed for reference.

However, we should note that Balaguer's response to Benacerraf's Problem is utterly abandoned by this characterisation of Hamkinsian Multiversism. First, on this account of the enterprise of set theory the response seems utterly unnecessary, it is the wrong kind of question to be asking about set-theoretic practice. Set theory here is rather viewed as an algebraic discipline not concerned with being 'about' any objects. Second, the characterisation also clearly undercuts the response in virtue of the acceptance of a thoroughgoing relativism with respect to metalogic. This makes it impossible to talk about 'reference via description' independent of assuming that one is already situated in some $V$, from the outside perspective it is not determinate what would even constitute a 'description' given the indeterminateness of formulae.

\section{Conclusion}

We have seen that Hamkins' Multiverse View appears to present a fascinating and elegant way of providing a background ontology for model-theoretic constructions. There appear to be two different aspects to the view, one ontological and one algebraic. The ontological interpretation of the view is seen to commit the Multiversist to a referential regress, vicious in virtue of the correlation of concepts with models. The problem is instructive for modern philosophical practice and shows that if one wishes to have an account of reference in set theory, a stock of notions taken to be absolute is required in order to secure a preferential class of models and delimit the multiverse. This is further seen with respect to metalogical definitions, as their satisfactory statement requires certain notions (such as finitude) to be absolutely understood. It is possible to maintain the Hamkinsian Multiverse perspective by viewing set theory as a fundamentally algebraic enterprise, but this is at the cost of a response to Benacerraf's Problem and a view of set theory on which we are concerned with ontology. While there are options open for the Hamkinsian, the moral is clear: if one wishes to have reference to mathematical objects, then relativism has to stop somewhere.

\section{References}

[Balaguer, 1998] Balaguer, M. (1998). Platonism and Anti-Platonism in Mathematics. Oxford University Press. 
[Benacerraf, 1973] Benacerraf, P. (1973). Mathematical truth. The Journal of Philosophy, 70(19):661-679.

[Feferman, 2011] Feferman, S. (2011). Is ch a definite problem? available from http://math.stanford.edu/ feferman/papers.html, accessed 13 April 2014.

[Field, 2003] Field, H. (2003). Do we have a determinate conception of finiteness and natural number? In Schirn, M., editor, The Philosophy of Mathematics Today. Clarendon Press.

[Foreman, 2010] Foreman, M. (2010). Ideals and generic elementary embeddings. In Kanamori, A. and Foreman, M., editors, Handbook of Set Theory, pages 885-1147. Springer.

[Gitman and Hamkins, 2011] Gitman, V. and Hamkins, J. D. (2011). A natural model of the multiverse axioms. Preprint. arXiv:1104.4450 [math.LO].

[Gödel, 1947] Gödel, K. (1947). What is cantor's continuum problem? In Kurt Gödel; Collected Works Vol. II. Oxford University Press.

[Hamkins, 2012a] Hamkins, J. D. (2012a). Pluralism in set theory: Does every mathematical statement have a definite truth value? In Philosophy Colloquium: CUNY Graduate Center.

[Hamkins, 2012b] Hamkins, J. D. (2012b). The set-theoretic multiverse. The Review of Symbolic Logic, 5(3):416-449.

[Hamkins and Loewe, 2008] Hamkins, J. D. and Loewe, B. (2008). The modal logic of forcing. Transactions of the American Mathematical Society, 360(4):1793-1817.

[Hamkins and Seabold, 2012] Hamkins, J. D. and Seabold, D. E. (2012). Wellfounded boolean ultrapowers as large cardinal embeddings. arXiv.

[Isaacson, 2011] Isaacson, D. (2011). The reality of mathematics and the case of set theory. In Noviak, Z. and Simonyi, A., editors, Truth, Reference, and Realism. Central European University Press.

[Jech, 2002] Jech, T. (2002). Set Theory. Springer.

[Koellner, 2013] Koellner, P. (2013). Hamkins on the multiverse. In Koellner, P., editor, Exploring the Frontiers of Incompleteness.

[Kunen, 1980] Kunen, K. (1980). Set Theory: An Introduction to Independence Proofs. Elsevier.

[Maddy, 1988] Maddy, P. (1988). Believing the axioms. i. The Journal of Symbolic Logic, 53(2):481-511.

[Maddy, 2011] Maddy, P. (2011). Defending the Axioms. Oxford University Press.

[Martin, 2001] Martin, D. (2001). Multiple universes of sets and indeterminate truth values. Topoi, 20(1):5-16.

[McGee, 1997] McGee, V. (1997). How we learn mathematical language. The Philosophical Review, 106(1):35-68.

[Meadows, 2013] Meadows, T. (2013). What can a categoricity theorem tell us? The Review of Symbolic Logic, 6:524-544. 
[Potter, 2004] Potter, M. (2004). Set Theory and its Philosophy: A Critical Introduction. Oxford University Press.

[Shapiro, 1991] Shapiro, S. (1991). Foundations without Foundationalism: A Case for Second-order Logic. Oxford University Press.

[Steel, 2014] Steel, J. (2014). Gödel's program. In Kennedy, J., editor, Interpreting Gödel. Cambridge University Press.

[Zermelo, 1930] Zermelo, E. (1930). On boundary numbers and domains of sets. In Ewald, W. B., editor, From Kant to Hilbert: a source book in Mathematics, volume 2, pages 1208-1233. OUP. 